because developmental studies have shown that lampreys and hagfishes are more closely related to each other than was previously imagined $^{7}$, it could also represent an animal close to the origin of cyclostomes before the lampreyhagfish divergence. That would mean that early cyclostomes exhibited a tremendous range of morphological variety. Indeed, the details of the early divergence of cyclostomes, which has been inferred to have occurred 390 million years ago or before ${ }^{8}$, remain unclear. Perhaps even weirder fossil vertebrates remain to be dug up.

Shigeru Kuratani and Tatsuya Hirasawa are in the Evolutionary Morphology Laboratory, RIKEN, Kobe, Hyogo 650-0047, Japan. e-mails:saizo@cdb.riken.jp; hirasawa@cdb.riken.jp

1. Traquair, R. H. Ann. Mag. Nat. Hist. 6th ser. 6, 479-486 (1890)
2. Johnson, R. G. \& Richardson, E. S. Fieldiana Geol. 12, 119-149 (1969).

3. McCoy, V. E. et al. Nature 532, 496-499 (2016)

4. Clements, T. et al. Nature 532, 500-503 (2016)

5. Richardson, E. S. Science 151, 75-76 (1966).

6. Janvier, P. Early Vertebrates (Oxford Univ. Press, 1996).

7. Oisi, Y., Ota, K. G., Fujimoto, S. \& Kuratani, S. Nature 493, 175-180 (2013).

8. Kuraku, S. \& Kuratani, S. Zool. Sci. 23, 1053-1064 (2006).

This article was published online on 13 April 2016.

\section{Four neutrons together momentarily}

\section{A system of four neutrons known as the tetraneutron is a hypothetical state in nuclear physics. The report of evidence for the fleeting existence of this state has implications for research into neutron stars.}

\section{CARLOS A. BERTULANI \& VLADIMIR ZELEVINSKY}

A tomic nuclei are composed of protons and neutrons, generically known as nucleons. These are not genuine elementary particles because they contain quarks and gluons, which interact with each other through the strong force (one of the four fundamental forces of nature). The strong interaction has subtle properties, with the most unsettling one being that quarks and gluons are never free, only confined within nucleons. Theorists continue to struggle to find exact solutions for various states of the highly complex quark-gluon systems, and to explain the nucleon-nucleon force that extends beyond

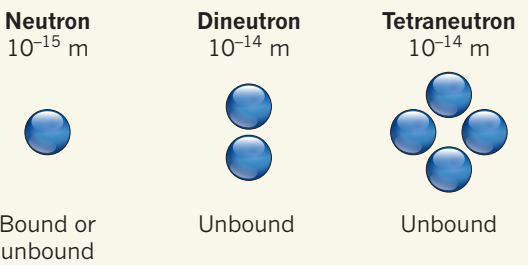

the confinement region. One long-sought state is the four-neutron system known as the tetraneutron, which has no electric charge. Writing in Physical Review Letters, Kisamori et al. ${ }^{1}$ present evidence for the existence of such a state.

In the authors' experiment, strongly bound a-particles (composed of two protons and two neutrons, and therefore identical to helium-4 nuclei) in liquid helium $-4\left({ }^{4} \mathrm{He}\right)$ are used as a target for an incident beam of helium $-8\left({ }^{8} \mathrm{He}\right.$, the 'projectile nucleus'). ${ }^{8} \mathrm{He}$ has two protons and six neutrons, and is produced in nuclearfragmentation reactions in which oxygen-18 hits a beryllium target. The reaction between ${ }^{8} \mathrm{He}$ and ${ }^{4} \mathrm{He}$ is an appropriate choice for generating tetraneutrons, because the four 'extra' neutrons in ${ }^{8} \mathrm{He}$ are weakly bound and

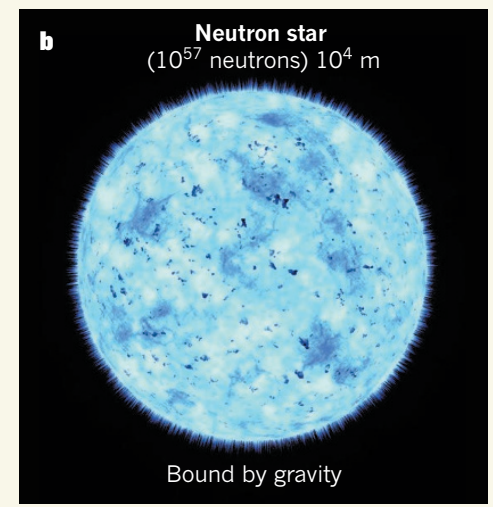

can be easily transformed in the interaction with ${ }^{4} \mathrm{He}$.

The authors observed that the ${ }^{8} \mathrm{He}$ projectile exchanges two units of charge with the ${ }^{4} \mathrm{He}$ target and becomes a beryllium- 8 nucleus $\left({ }^{8} \mathrm{Be}\right.$, four protons and four neutrons), the energy of which was measured with high precision. Because of charge conservation, the two protons in the target ${ }^{4} \mathrm{He}$ nucleus are substituted by neutrons, momentarily generating a fourneutron system in a quasi-bound state. This lasts only a few multiples of $10^{-22}$ seconds, after which it disassembles into free neutrons. This short-lived state appears as a bump in the energy spectrum of the ${ }^{8} \mathrm{Be}$ nucleus that emerges from the reaction.

Nuclear forces are essentially identical between all nucleons, whether they are protons or neutrons. So it might seem strange that the tetraneutron is not bound but that the a-particle of two protons and two neutrons is strongly bound, despite the additional electrical repulsion between protons. The explanation is based on the Pauli exclusion principle, which forbids two identical nucleons from occupying the same quantum state. In the a-particle, all four particles can be in the same state because the two protons have opposite spins, as does the pair of neutrons, so that all four nucleons are different. But for four neutrons, only one pair can be in the lowest-energy state, forcing the second pair into a state of higher energy, thus making the tetraneutron unstable.

By applying the principle of energy conservation to the studied nuclear reaction, Kisamori and colleagues infer that the tetraneutron system has an internal excitation energy of about 0.8 million electronvolts $(\mathrm{MeV})$; the excitation energy is the difference between the tetraneutron mass and the mass of four free neutrons. If this quantity were less than zero, the system would be bound. For the observed tetraneutron it is positive, making it an unbound system that exists for a short time before it decays into free neutrons. The statistical error $( \pm 0.65 \mathrm{MeV})$ and systematic error $( \pm 1.25 \mathrm{MeV})$ in the experiment are large, but the case for the existence of the tetraneutron is compelling. The width of the bump in the ${ }^{8} \mathrm{Be}$ energy spectrum is about $2.6 \mathrm{MeV}$, and this energy uncertainty suggests that the state will eventually decay to another quantum state.

The hunt for the tetraneutron has been going on for more than half a century, and
Figure 1 | Neutron systems. a, Neutrons have a radius of about one femtometre $\left(10^{-15} \mathrm{~m}\right)$, and can be either bound in a nucleus or free (although unbound neutrons decay within about 15 minutes). Dineutrons, composed of two unbound neutrons, are ten times larger and unstable. Kisamori and co-workers ${ }^{1}$ report $^{2}$ evidence for a tetraneutron (a system of four neutrons) that exists in a resonant state for about $10^{-22}$ seconds before dissociating into free neutrons. $\mathbf{b}$, If the existence of the tetraneutron state is confirmed, it will help to clarify nuclear interactions in few-nucleon systems, and possibly even in neutron stars. 
experimentalists have announced the state's discovery before. In 2002, one collaboration claimed to have found a bound tetraneutron ${ }^{2}$ in an experiment based on the detection of neutron clusters formed by fragmentation of beryllium-14 projectiles. But the result remains unconfirmed, and theorists quickly showed that, based on the best knowledge of the nucleon-nucleon interactions and other $\operatorname{arguments}^{3,4}$, the existence of a bound tetraneutron was nearly impossible.

However, theorists could not rule out the existence of a tetraneutron as a short-lived 'resonant' state on the basis of a dineutrondineutron structure ${ }^{3,4}$. The dineutron state is formed by two neutrons, and is not stable. It is known as a virtual state: if its energy were reduced by $66 \mathrm{keV}$, then the dineutron system would become bound. Decades earlier, it had been proposed ${ }^{5}$ that dineutrons can become bound in the presence of additional nucleons; this mechanism is responsible for the properties of some bound nuclei that have a neutron excess, such as lithium-11, in which a pair of external neutrons forms a remote halo around the core of lithium-9.

The tetraneutron cannot form an atomic nucleus because it is charge neutral and therefore cannot hold electrons. But there is an intimate relationship between the tetraneutron structure and theoretical studies of neutron stars (Fig. 1), in which neutrons are compressed to densities more than $10^{14}$ times that of water ${ }^{6}$. They are prevented from imploding by an outward pressure that is generated by the nucleon-nucleon interaction and other quantum-mechanical effects.

Nuclear physicists hope to develop a full understanding of how quarks and gluons inside nucleons generate nucleon-nucleon forces, and how many-body objects evolve to form complex structures such as the uranium nucleus and neutron stars. This is a formidable task, with well-understood parts but also many missing links. If Kisamori and co-workers' report of the tetraneutron state is confirmed, even as a short-lived resonance, it will add another structure to the nuclear chart that will help to improve our understanding of the nuclear interaction.

Carlos A. Bertulani is in the Department of Physics and Astronomy, Texas A\&M University-Commerce, Commerce, Texas 75429-3011, USA. Vladimir Zelevinsky is in the Department of Physics and Astronomy, Michigan State University, East Lansing, Michigan 48824-1321, USA.

e-mails: carlos.bertulani@tamuc.edu; zelevinsky@nscl.msu.edu

1. Kisamori, K. et al. Phys. Rev. Lett. 116, 052501 (2016).

2. Marqués, F. M. et al. Phys. Rev. C 65, 044006 (2002).

3. Bertulani, C. A. \& Zelevinsky, V. J. Phys. G 29, 2431-2437 (2003).

4. Pieper, S. C. Phys. Rev. Lett. 90, 252501 (2003).

5. Migdal, A. B. Sov. J. Nucl. Phys. 16, 238-241 (1973).

6. Shapiro, S. L. \& Teukolsky, S. A. Black Holes, White Dwarfs, and Neutron Stars (Wiley, 1983).

This article was published online on 6 April 2016.

\section{Fault tolerance in the brain}

\section{If stored information is erased from neural circuits in one brain hemisphere in mice, the lost data can be recovered from the other. This finding highlights a safeguarding mechanism at work in the brain. SEE ARTICLE P.459}

\section{BYRON M. YU}

W hen we send an e-mail or save a file on our hard drives, information can be lost, owing to dropped data packets or corrupted bits. We typically do not notice such failures because systems are designed with built-in mechanisms to restore the lost data. Dropped packets are retransmitted, and multiple copies of data are saved. The brain also stores and transmits information is it, too, fault-tolerant? In this issue, Li et al. ${ }^{1}$ (page 459) report the perturbation of brain activity to erase stored information in mice. They discover that the lost information can be rapidly restored by an unperturbed brain region.

The brain can reorganize itself to restore function after certain types of injury ${ }^{2}$, but this type of fault tolerance typically takes place over weeks. By contrast, many everyday brain functions, such as putting a name to the face of an acquaintance or hitting a tennis ball, take place on a timescale of seconds or less. Does a faulttolerance mechanism also operate in neural circuits over these shorter timescales?

$\mathrm{Li}$ et al. investigated whether regions present in each of the brain's two hemispheres might act together to produce a rapid back-up system for stored information - a mechanism

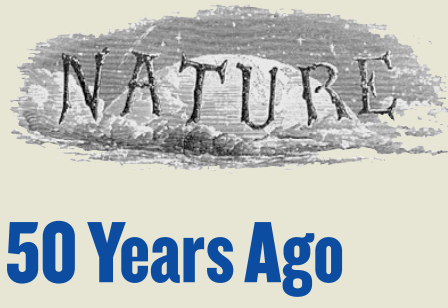

Hypnotic Susceptibility. By Ernest R. Hilgard - A large number of studies designed to investigate various hypnotic phenomena have been carried out by Ernest Hilgard and his co-workers on a considerable number of college students during the past eight years. Individual differences in 'hypnotizability' have been a major area of interest and in the course of their investigations several scales were developed for the quantitative assessment of hypnotic susceptibility ... There are three general purpose scales and a scale for yielding profiles of hypnotic ability. Convincing statistical evidence is given concerning their validity and reliability ... The latter part of the book is concerned with the relation of hypnotic susceptibility to a number of personality variables ... Although some significant correlations do emerge, they are insufficient to characterize the hypnotizable person clearly.

From Nature 30 April 1966

100 Years Ago

The large meteors which passed over Northern America on February 9, 1913, presented some unique features. The length of their observed flight was about 2600 miles, and they must have been moving in paths concentric, or nearly concentric, with the earth's surface, so that they temporarily formed new terrestrial satellites .. The meteors were last seen from the Bermuda Islands ... I have since made efforts to obtain further observations from seafaring men through the medium of the Nautical Magazine, and have succeeded in procuring data which prove that the meteors were observed during a course of 5500 miles from about lat. $51^{\circ} \mathrm{N}$., long. $107^{\circ} \mathrm{W}$., to lat. $5 \frac{1}{2}{ }^{\circ} \mathrm{S}$., long. $32 \frac{1}{2}{ }^{\circ} \mathrm{W}$.

From Nature 27 April 1916 